\title{
En defensa de la expansión internacional de Actas Urológicas Españolas
}

\author{
J.A. Lancina Martín \\ Servicio de Urología. Complejo Hospitalario Universitario Juan Canalejo. A Coruña.
}

Actas Urol Esp 2005; 29 (4): 345-348

$\mathrm{D}$ esde su fundación en el año 1977, la revista Actas Urológicas Españolas, órgano oficial de difusión de la Asociación Española de Urología, ha venido siguiendo una línea ascendente en cuanto a su difusión y calidad científica gracias al esfuerzo de todos los urólogos españoles, que han contribuido regularmente con la publicación de sus artículos, y particularmente de los directores que ha tenido la revista en sus diferentes etapas, el Dr. Alférez Villalobos, el Dr. Leiva Galvis y el Dr. Carballido Rodríguez. Durante este período de tiempo ha sido posible conseguir logros trascendentales como la inclusión de la revista en Medline, la principal base de datos gratuita de biomedicina en la red. También cabe destacar el acuerdo con la revista European Urology, órgano oficial de difusión de la Asociación Europea de Urología, para la publicación de los artículos de mayor calidad publicados en Actas Urológicas Españolas. Otro logro significativo ha sido la inclusión de la revista en el Índice Bibliográfico Español en Ciencias de la Salud (IBECS), impulsado por la Biblioteca Nacional de Ciencias de la Salud del Instituto Carlos III, para el desarrollo de la Biblioteca Virtual de la Salud (BVS) en colaboración con la Biblioteca Regional de Medicina de la Organización Panamericana de la Salud perteneciente a la Organización Mundial de la Salud (OPS/OMS), con el objetivo de facilitar la difusión de revistas científicas en lengua española. Como consecuencia de esta integración pudo ser incluida la revista en el Scientific Electronic Library on Line (SCIELO), una base de datos de revistas electrónicas con texto completo. Precisamente un avance muy notable en la difusión internacional de la revista fue el inicio de la edición electrónica (AEU on line), que ofrece de forma gratuita todos los artículos publicados a texto completo.
No hay duda de que estos logros han sido notables, pero es posible poner en marcha mecanismos que permitan mejorar la situación actual. Cualquier proceso de mejora debe perseguir al menos dos objetivos: aumentar la difusión y reconocimiento internacional de la revista y, en segundo lugar, mejorar la calidad científica de los artículos publicados. Como es sabido, ambos objetivos están intimamente relacionados, de forma que cualquier avance que se produzca en uno repercute positivamente sobre el otro. La experiencia seguida por otras publicaciones biomédicas nos ha mostrado lo determinante que es conseguir la más amplia difusión. Son varios los caminos que podemos seguir, pero sin duda la inclusión en los índices biomédicos más influyentes y el uso de la lengua inglesa han demostrado ser los factores más influyentes.

Un hecho incuestionable es aquel que se refiere al aplastante predominio e influencia de la lengua inglesa en la producción científica biomédica. Además de esto, los instrumentos y bases de datos que ejercen la organización, control y seguimiento de esta actividad científica, entre los que cabe destacar el Science Citation Index (SCI), que incluye las revistas cuyo factor de impacto (FI) publica el Journal Citation Reports (JCR), se adaptan perfectamente a los requerimientos del uso del inglés y, por ende, de la comunidad científica de influencia anglosajona. Producto de esta situación cabe destacar que solamente 20 revistas de países hispanos han conseguido ser incluidas en el SCI, de las cuales 14 están editadas en España, pero ninguna de ellas dedicada a enfermedades urológicas, y con un FI máximo de 1,88 para Histology and Histopathologhy correspondiente al año 2002 (Tabla 1). Por otra parte, solamente 35 revistas españolas están indexadas 
Tabla 1

Factor de impacto (FI) de revistas de medicina hispanoamericanas

\begin{tabular}{|c|c|c|}
\hline Revista & FI* & País \\
\hline Histology and Histopathology & 1,88 & España \\
\hline Revista Española de Cardiología & 0,94 & España \\
\hline Medicina Clínica & 0,85 & España \\
\hline Journal of Physiology and Biochemistry & 0,70 & España \\
\hline Revista Española de Enfermedades Digestivas & 0,59 & España \\
\hline Drugs News \& Perspectives & 0,52 & España \\
\hline Nefrología & 0,51 & España \\
\hline \multicolumn{3}{|l|}{ Methods and Findings in Experimental and } \\
\hline Clinical Pharmacology & 0,49 & España \\
\hline Drug Future & 0,39 & España \\
\hline Revista Clínica Española & 0,34 & España \\
\hline Revista de Investigación Clínica & 0,32 & Méjico \\
\hline Revista Médica de Chile & 0,29 & Chile \\
\hline Revista de Neurología & 0,29 & España \\
\hline Drugs of Today & 0,26 & España \\
\hline Actas Españolas de Psiquiatría & 0,26 & España \\
\hline Medicina & 0,25 & Argentina \\
\hline Neurocirugía & 0,24 & España \\
\hline Archivos Latinoamericanos de Nutrición & 0,24 & Venezuela \\
\hline Revista Ecuatoriana de Neurología & 0,15 & Ecuador \\
\hline Acta Bioquímica Clínica Latinoamericana & 0,11 & Argentina \\
\hline
\end{tabular}

*FI adjudicado por el Institute for Scientific Information (año 2002) fuerza y, por ende, su difusión y su calidad. La edición en lengua inglesa, aunque no imprescindible, sería un factor determinante por el dominio que tiene esta lengua en la comunidad científica internacional. Finalmente la mejoría del FI permitiría incluir nuestra revista en el SCI.

En el momento actual, Actas Urológicas Españolas está incluida en las bases de datos del IME, Medline, Embase/Excerpta Medica, Cuiden, IBECS y SCIELO, pero también debería conseguirse su inclusión en MedBio y SCI. Sería también deseable conseguir que los artículos de la revista que aparezcan en Medline pudieran ser consultados en texto completo mediante la creación de un hipervínculo, en el que además debería constar la condición de acceso gratuito. Esto no sería difícil de conseguir teniendo en cuenta que ya está disponible una edición electróni- en la base de datos Medline, lo que representa solamente el $\mathbf{1 7 , 5} \%$ del total de las editadas en España. En consecuencia, la presencia e influencia internacional de la producción científica procedente de los países hispanos y la editada en lengua española resulta ser muy inferior a la que realmente le correspondería por su calidad científica. El desinterés resultante de los autores hispanos provoca un desplazamiento de sus artículos de mayor calidad hacia las revistas publicadas en inglés con mejor FI.

El cambio de esta situación está plagado de enormes dificultades, pero son varios los senderos que pueden ayudar a conseguirlo. Entre ellos, cabe citar la creación de bases de datos alternativos al SCI que favorezcan la inclusión de revistas en español. Se debe reconocer que la creación del IBECS ha sido un paso muy importante para conseguir este objetivo. Otro camino sería la integración entre varias revistas ya existentes, que representen o no a sociedades científicas, de un mismo país o de varios, lo que potenciaría su ca de la revista con estas características. La experiencia que otras revistas españolas han tenido con esta iniciativa, como la Revista Española de Cardiología, ha resultado enormemente exitosa para su difusión, que además se ha visto incrementado por estar disponible una edición en inglés (free full english text).

Un objetivo muy ambicioso sería poder mejorar el FI para permitir que nuestra revista pudiera ser incluida en el SCI. El FI se calcula estableciendo la relación entre las citas bibliográficas que en un año reciben los artículos publicados en una revista en los dos años anteriores. Por tanto, el FI viene a medir la frecuencia con la que una revista ha sido citada en un año concreto, y por ello constituye un indicador de la influencia que sus artículos tienen en la comunidad científica, y es también un índice indirecto de la confianza que los investigadores ponen en ella. Este índice, con el tiempo, se ha convertido en un indicador de calidad, prestigio y difusión internacional para medir la calidad de la investigación 
realizada. En la actualidad, solamente 23 revistas de contenido urológico, general o específico, están incluidas en el SCI, ninguna de ellas editadas en lengua española ni provenientes de países hispanoparlantes (Tabla 2). La mayoría de estas revistas están respaldadas por una o más sociedades científicas y escritas en inglés. Solamente dos revistas están editadas en una lengua diferente, Urologe A en alemán y Progres en Urologie en francés. Además otras dos revistas se editan en inglés y simultáneamente en otra lengua, Annales d'Urologie en francés y Aktuelle Urologie en alemán. De las sociedades urológicas patrocinadoras de las revistas, 4 son de carácter internacional, 7 supranacional y 3 nacional.
Para hacer una estimación del impacto actual que tiene Actas Españolas de Urología puede servir de referencia el FI que, para las revistas médicas españolas, calcula el Instituto de Historia de la Ciencia y Documentación (IHCD), centro mixto de la Universidad de Valencia y el Consejo Español de Investigaciones Científicas (CSIC), en un proyecto respaldado por el Ministerio de Educación. Esta entidad utiliza los mismos criterios del Institute for Scientific Information para obtener el FI de las revistas incluidas en el JCR. Para el año 2002, nuestra revista obtiene un FI de 0,186 que la situaría solamente por encima de Annales d'Urologie y Aktualle Urologie, entre todas las revistas urológicas actualmente incluidas en este indicador (Tabla 2).

Tabla 2

Factor de impacto (FI) de revistas de Urología

\begin{tabular}{|c|c|c|c|c|}
\hline Revista & $\mathbf{F I}^{*}$ & Sociedad editora & Pais & Idioma \\
\hline Prostate & 3,167 & - & EE. UU. & Inglés \\
\hline Journal of Urology & 3,029 & American Urological Association & EE. UU. & Inglés \\
\hline Neurourology and Urodynamics & 2,560 & International Continence Society & EE. UU. & Inglés \\
\hline International Journal of Impotence Research & 2,544 & International Society for Sexual and Impotence Research & Reino Unido & Inglés \\
\hline Urology & 2,506 & Societé Internationale d'Urologie & EE. UU. & Inglés \\
\hline The Urologic Clinics of North America & 2,340 & - & EE. UU. & Inglés \\
\hline European Urology & 1,889 & European Association of Urology & Paises Bajos & Inglés \\
\hline World Journal of Urology & 1,679 & - & EE. UU. & Inglés \\
\hline \multirow[t]{2}{*}{ British Journal of Urology International } & \multirow[t]{2}{*}{1,640} & British Association of Urological Surgeons y Urological & & \\
\hline & & Society of Australasia & Reino Unido & Inglés \\
\hline $\begin{array}{l}\text { International Uroginecology Journal and Pelvic } \\
\text { Floor Dysfunction }\end{array}$ & 1,429 & $\begin{array}{l}\text { International Association of Uroginecology y Sociedad Mexicana } \\
\text { de Uroginecologia }\end{array}$ & Reino Unido & Inglés \\
\hline Molecular Urology & 1,310 & - & EE. UU. & Inglés \\
\hline Urological Research & 1,110 & - & EE. UU. & Inglés \\
\hline Journal of Endourology & 1,077 & Endourological Society y otras sociedades de Endourologia y ESWL & EE. UU. & Inglés \\
\hline Scandinavian Journal of Urology and Nephrology & 0,860 & $\begin{array}{l}\text { Scandinavian Association of Urology y otras sociedades urológicas } \\
\text { noreuropeas }\end{array}$ & Noruega & Inglés \\
\hline Asian Journal of Andrology & 0,836 & Asian Society of Andrology & China & Inglés \\
\hline $\begin{array}{l}\text { Urologic Oncology - Seminars and Original } \\
\text { Investigations }\end{array}$ & 0,818 & Society of Urologic Oncology & EE. UU. & Inglés \\
\hline Urologia Internationalis & 0,500 & - & Suiza & Inglés \\
\hline Urologe A & 0,496 & Deutschen Geselschaft fur Urologie & Alemania & Alemán \\
\hline Prostate Cancer and Prostatic Disease & 0,458 & - & Reino Unido & Inglés \\
\hline International Journal of Urology & 0,448 & Japanese Urological Association & Japón & Inglés \\
\hline Progres en Urologie & 0,436 & $\begin{array}{l}\text { Association Francaise d'Urologie, Association des Urologues du } \\
\text { Quebec y Societé Belge d'Urologie }\end{array}$ & Francia & Francés \\
\hline Actas Urológicas Españolas & 0,186 & Asociación Española de Urología & España & Español \\
\hline Annales d'Urologie & 0,157 & - & Francia & Francés / Inglés \\
\hline Aktuelle Urologie & 0,089 & - & Alemania & Alemán / Inglés \\
\hline
\end{tabular}

*FI adjudicado por el Institute for Scientific Information (año 2002), con adaptación para Actas Urológicas Españolas por el IHBD. 
Para conseguir aumentar la difusión internacional de nuestra revista, y su inclusión en el índice SCI, deberíamos buscar el perfil que más se aproxime al de las revistas incluidas en esta base. El perfil más común se caracteriza por el patrocinio de varias sociedades científicas, su carácter supranacional y uso del inglés. Si pretendemos buscar la integración con las revistas de otras sociedades urológicas, está claro que por razones históricas, culturales y por mantener una lengua común, esta confluencia debería hacerse con las sociedades urológicas hispanoamericanas $\mathrm{y}$, en última instancia, también con Portugal y Brasil. Un primer paso importante sería la fusión con la revista de la Confederación Americana de Urología, por la influencia que esta sociedad tiene en Latinoamérica. En cierto modo, esta vía ha sido la estrategia que se ha seguido en el Reino Unido y en Francia. La revista British Journal of Urology International está patrocinada por la Asociación Británica de Urología y la Sociedad Urológica de Australasia, mientras que la revista Progres en Urologie está patrocinada por la Asociación Francesa de Urología, la Asociación de Urólogos de Québec y la Sociedad Belga de Urología. En Europa, solamente la Sociedad Alemana de Urología se mantiene en solitario patrocinando la revista Urologe A, pero obligada a ello por carecer de una comunidad germanoparlante y también a cambio de conseguir unos resultados muy discretos de FI.

En cuanto al idioma, sin duda alguna la lengua española deberá seguir manteniéndose pero sería oportuno incluir también una edición de la revista electrónica en inglés, lo que contribuiría enormemente a su difusión como ha ocurrido con las revistas en otras lenguas que han seguido esta directriz. La posibilidad de que se unieran a este proyecto Portugal y Brasil, debería contemplar lógicamente también el uso oficial del portugués.

Además de todas estas consideraciones, deberían de ponerse en práctica otras actuaciones como campañas de marketing para dar a conocer la revista en los ámbitos mayores posibles. Se procuraría, por tanto, un aumento de distribución de la revista, especialmente a bibliotecas e instituciones biomédicas, tanto a nivel nacional como internacional, poniendo especial atención en Hispanoamérica y Europa. También deben ser considerados los comunicados de prensa sobre los artículos publicados con un posible mayor atractivo para la población, conferencias de prensa sobre los artículos de mayor repercusión, inclusión de propaganda en medios de comunicación biosanitarios y presencia con stands en reuniones científicas nacionales e internacionales, en especial aquellas dirigidas a sectores interesados en las enfermedades genitourinarias, etc. Tampoco se deben descuidar aquellos aspectos que puedan hacer más atractiva la revista para los potenciales lectores. Se puede mejorar el diseño, formato y distribución interna, como la misma calidad del papel y la impresión para mejorar su lectura. Se pueden incluir nuevas secciones que marquen el camino de la actualidad, invitación a escribir artículos y editoriales a profesionales con prestigio, independientemente de su nacionalidad y país de origen, edición de números monográficos, potenciación de los artículos originales, aumento de la cuantía de los trabajos premiados para incentivar la publicación, optimización de todos los procesos editoriales con el fin de reducir los tiempos de evaluación, decisión y publicación, etc.

Debe entenderse que cualquier cambio que se produzca debe partir del punto en que nos encontramos, y debe ser ampliamente aceptado por los comités de la revista, por la Junta Directiva de la Asociación Española de Urología y por los socios que la componen. Como condición necesaria, se debe mantener indemne la personalidad propia de la Asociación Española de Urología, en donde la nueva revista debería seguir siendo su órgano oficial de difusión, aunque claro está compartiendo también esta función con las otras sociedades científicas que pudieran adherirse a este proyecto, pero todo ello sin renunciar a que sea una herramienta cientifica útil y práctica con un amplio reconocimiento nacional e internacional.

Dr. J.A. Lancina Martín

Servicio de Urología

Complejo Hospitalario Univ. Juan Canalejo

Xubias de Arriba, 84

15006 A Coruña 